\title{
Rejort of the Snowmass M6 Working Group On High Intensity Proton Sources
}

\author{
W. Chou (Fermílab), J. Wei (BNL)
}

August 10, 2001

Formal Report

Collider Accelerator Development Department

Spallation Neutron Source Project

Brookhaven National Laboratory

Brookhaven Science Associates

Upton, NY 11973

Under Contract No. DE-AC02-98CH10886 with the

United States Department of Energy 


\section{DISCLAIMER}

This report was prepared as an account of work sponsored by an agency of the United States Government. Neither the United States Government nor any agency thereof, nor any employees, nor any of their contractors, subcontractors or their employees, makes any warranty, express or implied, or assumes any legal liability or responsibility for the accuracy, completeness, or any third party's use or the results of such use of any information, apparatus, product, or process disclosed, or represents that its use would not infringe privately owned rights. Reference herein to any specific commercial product, process, or service by trade name, trademark, manufacturer, or otherwise, does not necessarily constitute or imply its endorsement, recommendation, or favoring by the United States Government or any agency thereof or its contractors or subcontractors. The views and opinions of authors expressed herein do not necessarily state or reflect those of the United States Government or any agency thereof. 


\section{BNL -52639 \\ FORMAL REPORT}

Report of the Snowmass M6 Working Group On High Intensity Proton Sources

W. Chou (Fermilab), J. Wei (BNL)

August 10, 2001 


\title{
Report of the Snowmass M6 Working Group on High Intensity Proton Sources*
}

\author{
Coriveners: W. Chou (Fermilab) and J. Wei (BNL)
}

August 10, 2001

Charge to the group: Several present and future high-energy physics facilities are based on high intensity secondary particle beams produced by high intensity proton beams. The group is to perform a survey of the beam parameters of existing and planned multi $\mathrm{GeV}$ high intensity proton sources and compare them with the requirements of high-energy physics users of secondary beams. The group should then identify areas of accelerator R\&D needed to achieve the required performance. This should include simulations, engineering and possibly beam experiments. The level of effort and time scale should also be considered.

Executive summary

\section{$\underline{\text { Outline }}$}

1. Introduction

2. Linac and transport lines

2.1 Ion source

2.2 Low-energy beam transport (LEBT) and radio frequency quadrupole (RFQ)

2.3 Medium-energy beam transport (MEBT)

2.4 Funneling

2.5 Accelerator architecture and structures

2.6 Superconducting RF linac

$2.7 \mathrm{RF}$ control

2.8 High-energy beam transport (HEBT) and ring-to-target beam transport (RTBT)

2.9 Space charge effects

2.10 Diagnostics

\section{Ring}

3.1 Lattice, aperture and corrections

3.2 Injection and extraction

3.3 Space charge and halo

3.4 Beam stability and impedance

3.5 Electron cloud

3.6 Beam loss, collimation and protection

3.7 Magnets and kickers

3.8 Power supplies

$3.9 \mathrm{RF}$

3.10 Beam loading and compensation

3.11 Diagnostics

3.12 Inductive inserts

3.13 Fixed field alternating gradient synchrotron (FFAG)

3.14 Induction synchrotron

4. List of participants

5. List of talks

* Website: http://www-bd.fnal.gov/icfa/snowmass/ 


\section{Executive Summary}

The U.S. high-energy physics program needs an intense proton source, a 1-4 MW Proton Driver (PD), by the end of this decade. This machine will serve as a stand-alone facility that will provide neutrino superbeams and other high intensity secondary beams such as kaons, muons, neutrons, and anti-protons (cf. E1 and E5 group reports) and also serve as the first stage of a neutrino factory (cf. M1 group report). It can also be a high brightness source for a VLHC.

Based on present accelerator technology and project construction experience, it is both feasible and cost-effective to construct a 1-4 MW Proton Driver. Two recent PD design studies have been made, one at FNAL and the other at the BNL. Both designed PD's for $1 \mathrm{MW}$ proton beams at a cost of about U.S. \$200M (excluding contingency and overhead) and both designs were upgradeable to $4 \mathrm{MW}$. An international collaboration between FNAL, BNL and KEK on high intensity proton facilities is addressing a number of key design issues. The superconducting (sc) RF cavities, cryogenics, and RF controls developed for the SNS can be directly adopted to save R\&D efforts, cost, and schedule. PD studies are also actively being pursued at Europe and Japan.

There are no showstoppers towards the construction of such a high intensity facility. Key research and development items are listed below ( \{\} indicates present status). Category $\mathrm{A}$ indicates items that are not only needed for future machines but also useful for improving the performance of existing machines; category B indicates items crucial for future machines and/or items currently underway.

1) $\mathrm{H}^{-}$source: development goals are to achieve a current of $60-70 \mathrm{~mA}\{35 \mathrm{~mA}\}$, duty cycle $6-12 \%\{6 \%\}$, emittance $0.2 \pi \mathrm{mm}-\mathrm{mrad} \mathrm{rms}$ normalized, and lifetime $>2$ months $\{20$ days $\}$. (A)

2) LEBT chopper: achieve rise time $<10 \mathrm{~ns}\{50 \mathrm{~ns}\}$. (B)

3) Study a 4-rod RFQ at $400 \mathrm{MHz}, 100 \mathrm{~mA}$, and $99 \%$ efficiency, HOM suppressed. (B)

4) MEBT chopper: achieve rise time $<2 \mathrm{~ns}\{10 \mathrm{~ns}\}$. (B)

5) Chopped beam dump: perform material study and engineering design for dumped beam power $>10 \mathrm{~kW}$. (A)

6) Funneling: (i) perform one-leg experiment at the RAL by 2006 with a goal of a oneleg current of $57 \mathrm{~mA}$; (ii) design deflector cavity for CONCERT. (all B)

7) Linac RF control: develop (i) a high performance HV modulator for long pulsed ( $>1 \mathrm{~ms}$ ) and $\mathrm{CW}$ operation; (ii) high efficiency RF sources (IOT, multi-beam klystron). (all A)

8) SC linac RF control: goal is to achieve control of RF phase error $<0.5^{\circ}$ and amplitude error $<0.5 \%$ \{presently $1^{\circ}, 1 \%$ for a warm linac\} (i) investigate the choice of RF source (number of cavities per RF source, use of high-power source); (A) (ii) perform a redundancy study for high reliability; (B) (iii) develop high performance RF control (feedback and feed-forward) during normal operation, tuning phases and off-normal operation (missing cavity), including piezo-electric fast feed-forward. (A)

9) Space charge: (i) compare simulation code ORBIT with machine data at FNAL Booster and BNL Booster; (ii) perform 3-D ring code bench marking including machine errors, impedance, and space charge (ORNL, BNL, SciDAC, PPPL). (all A) 
10) Linac diagnostics: develop (i) non-invasive (laser wire, ionization, fluorescent-based) beam profile measurement for $\mathrm{H}^{-}$; (ii) on-line measurement of beam energy and energy spread using time-of-flight method; (iii) halo monitor especially in sc environment; (iv) longitudinal bunch shape monitor. (all A)

11) SC RF linac: (i) obtain high gradients in an intermediate beta $(0.5-0.8)$ cavity; (A) (ii) develop spoke cavity for low beta $(0.17-0.34)$. (B)

12) Transport lines: develop (i) high efficiency collimation systems; (A) (ii) profile monitor and halo measurement; (A) (iii) energy stabilization by HEBT RF cavity using feed-forward to compensate phase-jitter. (B)

13) Halo: (i) continue LEDA experiment on linac halo and comparison with simulation; (ii) begin halo measurement in rings and comparison with simulation. (all B)

14) Ring lattice: study higher order dependence of transition energy on momentum spread and tune spread, including space charge effects. (B)

15) Injection and extraction: (i) develop improved foil (lifetime, efficiency, support); (A) (ii) experiment on the dependence of $\mathrm{H}^{\mathrm{P}}$ excited states lifetime on magnetic field and beam energy; (B) (iii) determine the efficiency of slow extraction systems. (A)

16) Electron cloud: (i) measure and simulate electron cloud generation (comparison of the measurements at CERN and SLAC on the interaction of few $\mathrm{eV}$ electrons with accelerator surfaces, investigation of angular dependence of SEY, machine and beam parameter dependence); (A) (ii) determine electron density in the beam by measuring the tune shift along the bunch train; (A) (iii) develop the theory for bunched beam instability that reliably predicts instability thresholds and growth rates; (A) (iv) investigate surface treatment and conditioning; (A) (v) study a fast, wide-band, active damping system at the frequency range of 50-800 $\mathrm{MHz}$. (B)

17) Ring beam loss, collimation, protection: (i) benchmark and validate code (STRUCT, $\mathrm{K} 2$, ORBIT); (A) (ii) produce an engineering design of collimator and beam dump; (A) (iii) experimentally study the efficiency of beam-in-gap cleaning; (A) (iv) perform bent crystal collimator experiment in RHIC; (B) (v) study collimation with resonance extraction. (B)

18) Ring diagnostics: (i) diagnose beam parameters during multi-turn injection; (ii) develop a circulating beam profile monitor covering a large dynamic range with turnby-turn speed; (iii) develop a method for fast, accurate non-invasive tune measurement. (all A)

19) Ring RF: develop (i) low frequency ( $\sim 5 \mathrm{MHz})$, high gradient ( $\sim \mathrm{MV} / \mathrm{m})$ burst mode $\mathrm{RF}$ systems; (B) (ii) a high gradient ( $50-100 \mathrm{kV} / \mathrm{m}$ ), low frequency (several $\mathrm{MHz}$ ) RF system with $50-60 \%$ duty cycle; (B) (iii) a high-voltage $(>100 \mathrm{kV})$ barrier bucket system; (B) (iv) transient beam loading compensation systems (e.g. for low-Q MA cavity). (A)

20) Ring magnets: (i) develop stranded conductor coil; (ii) study voltage-to-ground electrical insulation; (iii) study dipole/quadrupole tracking error correction. (all B)

21) Ring power supplies: develop (i) duatharmonic resonant power supplies; (ii) cost effective programmable power supplies. (all B)

22) Kicker: develop (i) stacked MOSFET modulator for DARHT and AHF to achieve rise/fall time <10-20 ns; (B) (ii) develop impedance reduction of lumped ferrite kicker for SNS. (A) 
23) Instability and impedance: (i) establish approaches for improved estimates of thresholds of fast instabilities, both transverse and longitudinal (including space charge and electron cloud effects); (ii) place currently-used models such as the broadband resonator and distributed impedance on a firmer theoretical basis; (iii) develop a method for impedance measurement based on coherent tune shifts $v s$. beam intensity, and instability growth rate $v s$. chromaticity, including that for flat vacuum chambers; (iv) develop new technology in feedback implementation. (all B)

24)FFAG: (i) perform 3-D modeling of magnetic fields and optimization of magnet profiles; (ii) develop wide-band RF systems; (iii) develop transient phase shift in high frequency RF structures; (iv) study the application of sc magnets. (all B)

25) Inductive inserts: (i) perform experiments at the FNAL Booster \& JHF3; (A) (ii) develop programmable inductive inserts; (B) (iii) develop inductive inserts which have large inductive impedance and very small resistive impedance; (B) (iv) perform theoretical analysis. (B)

26) Induction synchrotron: (i) study beam stability; (ii) develop high impedance, low loss magnetic cores. (all B) 


\section{Introduction}

The M6 working group had more than 40 active participants (listed in Section 4). During the three weeks at Snowmass, there were about 50 presentations, covering a wide range of topics associated with high intensity proton sources. The talks are listed in Section 5. This group also had joint sessions with a number of other working groups, including E1 (Neutrino Factories and Muon Colliders), E5 (Fixed-Target Experiments), M1 (Muon Based Systems), T4 (Particle Sources), T5 (Beam dynamics), T7 (High Performance Computing) and T9 (Diagnostics).

The M6 group performed a survey of the beam parameters of existing and proposed high intensity proton sources, in particular, of the proton drivers. The results are listed in Table 1 . These parameters are compared with the requirements of high-energy physics users of secondary beams in Working Groups E1 and E5. According to the consensus reached in the E1 and E5 groups, the U.S. HEP program requires an intense proton source, a 1-4 MW Proton Driver, by the end of this decade.

Table 1. Beam Parameters of Existing and Proposed Proton Sources

\begin{tabular}{|l|c|c|c|c|c|}
\hline \multicolumn{1}{|c|}{ Machine } & $\begin{array}{c}\text { Flux } \\
\left(10^{13} / \text { pulse }\right)\end{array}$ & $\begin{array}{c}\text { Rep Rate } \\
(\mathrm{Hz})\end{array}$ & $\begin{array}{c}\text { Flux } \\
\left(10^{20} / \text { year }\right)\end{array}$ & $\begin{array}{c}\text { Energy } \\
(\mathrm{GeV})\end{array}$ & $\begin{array}{c}\text { Power } \\
(\mathrm{MW})\end{array}$ \\
\hline Existing: & 2.5 & 50 & 125 & 0.8 & 0.16 \\
RAL ISIS & 7 & 0.5 & 3.5 & 24 & 0.13 \\
BNL AGS & 2.5 & 20 & 50 & 0.8 & 0.064 \\
LANL PSR & 0.5 & 7.5 & 3.8 & 8 & 0.05 \\
Fermilab MiniBooNE (*) & 3 & 0.5 & 1.5 & 120 & 0.3 \\
Fermilab NuMI & 4.8 & 0.17 & 0.8 & 400 & 0.5 \\
CERN CNGS & & & & & \\
Under construction: & 20 & 60 & 1200 & 1 & 2 \\
ORNL SNS & 32 & 0.3 & 10 & 50 & 0.75 \\
JHF 50 GeV & 8 & 25 & 200 & 3 & 1 \\
JHF 3 GeV & 3 & 15 & 45 & 16 & 1.2 \\
\hline Proton Driver proposals: & 10 & 15 & 150 & 16 & 4 \\
Fermilab Phase I & 10 & 2.5 & 25 & 24 & 1 \\
Fermilab Phase II & 20 & 5 & 100 & 24 & 4 \\
BNL Phase I & 23 & 50 & 1100 & 2.2 & 4 \\
BNL Phase II & 6.6 & 25 & 165 & 15 & 4 \\
CERN SPL (PDAC) & 10 & 50 & 500 & 5 & 4 \\
RAL 15 GeV (**) & & & & & \\
RAL 5 GeV (**) & 46.8 & 50 & 2340 & 1.334 & 5 \\
\hline Other proposals: & 234 & 50 & 12000 & 1.334 & 25 \\
Europe ESS (**) & - & CW & 62500 & 1 & 100 \\
Europe CONCERT & 3 & 0.04 & 0.03 & 50 & 0.003 \\
LANL AAA & & & & & \\
LANL AHF & & & & & \\
& & & & & \\
\end{tabular}

$\dagger 1$ year $=1 \times 10^{7}$ seconds.

(*) Including planned improvements.

(**) Based on 2-ring design. 
The M6 group has also identified areas of accelerator R\&D needed to achieve the required performance of a Proton Driver. These $R \& D$ items are divided into three categories:

- Category A includes those items that are not only needed by future machines but will also be useful for improving the performance of existing machines. Therefore, they have the highest priority.

- Category B is the R\&D work that is critical to future machines and/or is currently underway as part of established collaborations.

- Category $C$ lists other R\&D items that are necessary to future machines but may have to wait until more resources can be made available.

It should be pointed out that there are presently two pulsed mega-watt high intensity proton facilities under construction: the Spallation Neutron. Source (SNS) utilizing a superconducting RF linac, and the JAERI/KEK Joint Project (JHF) utilizing rapidcycling synchrotrons. Progress made on these projects can greatly benefit the design and development of high power Proton Drivers.

\section{Linac and transport lines}

\subsection{Ion source:}

Ion sources are critical elements in terms of beam quality (intensity - emittance stability) and reliability (sparks - life time). Their achievable performance has a strong influence on the design and cost of the whole linac.

High-current proton sources (up to $\sim 200 \mathrm{~mA}$ ) have been available for several decades for linacs used in nuclear and particle physics facilities. Nevertheless, the source technologies developed for low duty cycles (few $\times 10^{-3}$ ) lead to lifetime and reliability problems when increased duty factors are needed ( $5 \%$ up to $\mathrm{CW}$ ). More recent R\&D work done at Chalk River, Los Alamos and Saclay has demonstrated that the ECR type proton sources are ideal for this high duty factor range (I $>100 \mathrm{~mA}, \Delta \mathrm{I} / \mathrm{I}<1 \%, \varepsilon \sim 0.2 \pi$ mm-mrad rms normalized, duty cycle up to CW, life time $\sim 6$ months). R\&D efforts must be focused now on further emittance reduction ( $\varepsilon<0.1 \pi \mathrm{mm}$-mrad rms normalized) to relax the constraints on the RFQ and following structures and on beam current control for the commissioning and power ramping phases (intensity and pulse shape control).

High performance $H$ sources must be a high priority $R \& D$ subject. The $H$ sources used for accelerator operation have limited performance when high intensity, low beam noise, low emittance, long lifetime and high duty factor are all required simultaneously. At Fermilab, the goals for the $H$ source $R \& D$ are $I_{\max }=115 \mathrm{~mA}, \varepsilon \sim 0.25 \pi \mathrm{mm}$-mrad rms normalized, lifetime $=4$ months, duty cycle $=0.5 \%$. At the BNL, the parameters of the present $\mathrm{H}^{-}$source are $\mathrm{I}_{\max }=120 \mathrm{~mA}, \varepsilon \sim 0.37 \pi \mathrm{mm}$-mrad ms normalized, lifetime $=$ 2 months for $0.7 \%$ duty cycle operation. At $5 \%$ duty cycle and I $\sim 60 \mathrm{~mA}, \varepsilon<0.2 \pi \mathrm{mm}$ mrad rms normalized, the lifetime becomes less than one month. Efforts must be taken on all different types of $\mathrm{H}^{-}$sources (Penning, volume, ECR...) in order to achieve significant short- and long-term progresses. The SNS H source program, the Negative Ion Source (NIS) network supported by the European Community, and the intensive work being 
done in Japan and other countries serve as a good basis for a fruitful international collaboration. In particular, the R\&D on the ECR H source at both Saclay and LANL to achieve high duty factors and long lifetime should be strengthened. R\&D efforts should also be devoted to the control of the cesium flow.

\subsection{LEBT and RFQ:}

The two types of LEBT are magnetic (at LEDA, IPHI, BTA...) and electrostatic (at SNS). One of the most important issues in the magnetic LEBT is space-charge neutralization. A quantitative understanding is still lacking and is required for significant progress in this area. Another area of study is the identification of relevant diagnostics in the LEBT for characterization of the beam. This is intimately tied to the controls needed to match the beam into the RFQ once the proper set of diagnostics for beam characterization is in place. For a high duty factor ( $5 \%$ or more) $\mathrm{H}$ beam, another critical issue is the chopping structure of the beam. With a growing need to provide a higher duty factor, one would be forced to provide fewer particles in the gap i.e. a cleaner notch in the beam macro-pulse. This can only be achieved with a clear understanding of the pre-chopper physics in terms of neutralization time constant for a magnetic LEBT. For the electrostatic LEBT, the challenge is to reduce the time constant from $\sim 50 \mathrm{~ns}$ to $\sim 2 \mathrm{~ns}$. The proposed double $\alpha$ system at FNAL will be a positive step in this area.

Successful design and operation of high power RFQs have laid the foundation for high intensity $\mathrm{H}^{+} / \mathrm{H}^{-}$linacs. Such RFQs are in operation or being constructed across the globe: Los Alamos (LEDA, GTA...), Japan (BTA), France (IPHI), Korea (KOMAC), and LBL (SNS). They provide beams for very low duty factor all the way up to CW. The RFQ at CERN and LANL provide the highest peak currents. The CERN RFQ provides about $200 \mathrm{~mA}$ of peak current with a very low duty factor, while the LEDA RFQ at LANL produces $\sim 100 \mathrm{~mA}$ of $\mathrm{CW}$ beam. The above noted RFQs are all 4 -vane type that demand state of the art engineering. Another class of RFQs are 4-rod RFQs. The University of Frankfurt (UF) has been in the forefront of this technology. A large number of 4-rod RFQs built by UF are in operation around the world.

Although in many ways RFQs are a mature technology, specifically in terms of beam and RF physics understanding, two issues are worth pursuing. First is the viability of 4 rod RFQ for higher frequency i.e., $200 \mathrm{MHz}$ and above. This question has significant impact on the construction budget and schedule not to mention the relative ease of engineering implementation of the structure. The second topic that has a very critical impact on the operational reliability/availability issue is the relationship between sparking-down frequency and surface treatment and vacuum quality. The very stringent (few tens a day) spark-down requirement stipulated for some high power linac (ATW) applications makes this an important R\&D item.

\subsection{MEBT:}

A traveling wave type chopper for $\mathrm{H}$ beam at $750 \mathrm{keV}$ has been in operation for the last several decades at LANL and BNL. Another one is under construction at Los Alamos for the SNS project to chop $\mathrm{H}$ beam at $2.5 \mathrm{MeV}$. Though similar in physics concept, $\mathrm{RF}$ is handled somewhat differently in terms of hardware. Some questions still remain about 
the long- term degassing effect of the dielectric containing the meander-line. This should be answered by operational experience at the SNS. Not achieved yet, however, is the short rise/fall time of the pulser. The presently achieved number is $\sim 10 \mathrm{~ns}$. A short ( $2 \mathrm{~ns}$ or lower) time constant with relatively high voltage ( $1 \mathrm{kV}$ or higher) is necessary to eliminate the use of the anti-chopper that nearly doubles the length of the MEBT section. This seriously deteriorates the beam quality through the MEBT section thereby affecting the beam-performance down the linac. An RF R\&D effort is urgently needed in this area. Needed also is R\&D on diagnostics in the area of innovative emittance measurement technique(s) for the low energy, high power beam in the MEBT. This should help achieve better matching of the beam into the following linac structure, thereby greatly improving the overall performance of the linac in terms of beam loss.

The beam dump that accepts the chopped beam is another area where substantial mechanical, material, and RF R\&D effort is needed in the very near future. Dumps for pulsed high power linacs will be required to handle greater than $10 \mathrm{~kW}$ of beam power. There are at least three areas of concern: (1) material and cooling, (2) suppression of secondary electrons, and (3) material sputtering.

\subsection{Funneling:}

Working experience in this area is limited. Los Alamos pioneered this concept and demonstrated its feasibility in a one-leg experiment in the late ' 80 s to early '90s. Recently, RAL has proposed a one-leg experiment with $57 \mathrm{~mA}$ of beam current around the year 2006. Also noteworthy is the two beam-RFQ experiment performed at the University of Frankfurt during the late ' 90 s. It shares the same idea, i.e. merging of two identical beams in longitudinal phase space. However, this uses a continuous channel of the RFQ structure, whereas traditional funneling uses discrete elements.

Several proposed high-power designs (earlier versions of CW APT $200 \mathrm{MW}$ designs at Los Alamos, recent ESS and CONCERT designs in Europe) are based on funneling. The desirability of funneling at relatively higher energy (around $20 \mathrm{MeV}$ ) also means that work is needed to design a suitable structure between the first RFQ and the funnel. Funneling is of the same level of importance as a higher intensity $\mathrm{H}^{-}$ion source. Progress towards a higher intensity ion source has been slow so it is important that funneling be given the same level of priority in the R\&D effort as a higher intensity $\mathrm{H}$ ion source. These efforts have the goal of a higher power (greater than 2 or $3 \mathrm{MW}$ ) $\mathrm{H}^{-}$linac. Dedicated two-leg experiments are needed addressing the issues of: (a) effective emittance of the funneled beam and (b) properties of the ion-beams from the two legs in terms of intensity, and effective emittance and noise levels.

In addition to the early LANL RF-deflector cavity work, a new design capable of providing higher deflection angle at relatively higher energy $(20 \mathrm{MeV})$ has recently been developed jointly by the CEA-Saclay (France) and Protvino (Russia) groups for the CONCERT project.

\subsection{Accelerator architecture and structures:}

A typical $1-\mathrm{GeV}$ proton linac, suitable for average beam power up to several $\mathrm{MW}$, is a pulsed machine with a pulse length of about 1 millisecond, and a repetition rate in the 
range of 50 to $100 \mathrm{~Hz}$. The linac consists of three main sections: a radio frequency quadrupole linac or RFQ, intermediate-velocity accelerating structures, and high velocity structures. A DC injector delivers an unbunched $50-$ to $100-\mathrm{kV}$ beam with beam current in the range of a few tens of $\mathrm{mA}$ to the normal conducting RFQ. The RFQ provides strong periodic RF electric quadrupole focusing, and adiabatically bunches and accelerates the low-velocity beam to a few $\mathrm{MeV}$ of energy.

The RFQ is followed by the intermediate velocity structures which accelerate the beam within the velocity range of about $\beta=0.1$ to about 0.4 , corresponding to an output energy of about $100 \mathrm{MeV}$. The intermediate velocity structures may either be some type of normal-conducting drift-tube linac structures (e.g. DTL, SDTL, CCDTL or RFD) or superconducting structures such as a spoke resonator. The frequency of the RFQ and the intermediate velocity structures is typically in the range of about 200 to $400 \mathrm{MHz}$.

The intermediate velocity structures are followed by the high velocity structures, which may be normal-conducting coupled-cavity structures (SCL or ACL) or superconducting multi-cell elliptical cavities. The RF frequency of the high velocity structures is typically a few multiple of the intermediate velocity structures.

The superconducting RF (SRF) linacs for both the intermediate and high velocities are comprised of individual sections each with identical cavities and cryomodules. Either quadrupoles or solenoids provide the transverse focusing for the intermediate- and highvelocity structures. For superconducting sections these may either be normal conducting magnets outside the cryomodules or superconducting magnets within the cryomodules. Typically either singlet FODO or doublet lattices may be used; the choice depends on the overall required focusing strength and the space available for the individual lenses.

\subsection{Superconducting $\mathbb{R F}$ linac:}

Superconducting RF linacs provide several advantages over normalconducting linacs, which include reduction in RF power dissipation by four to five orders of magnitude, higher accelerating gradients, and larger bore radii, which become affordable without the penalty of large increases in RF power as in normatconducting linacs. The RF power reduction lowers both the capital costs for RF power equipment and the operating ac power costs. Higher accelerating gradients reduce the linac length. Larger bore radii relax the alignment, beam steering, and beam-matching tolerances, and reduce the beam loss and associated induced radioactivity. The number of cells per superconducting cavity is usually less than 10 , which is much smaller than for normal conducting cavities. The smaller number of cells per cavity allows for a broader velocity acceptance so that the intermediate- and high-velocity range can be covered with just a few distinct cavity beta values.

The $Q$ values for superconducting cavities are fairly high $\left(10^{9}\right.$ or higher), even when loading from the power couplers is included. This, together with the requirement that in a proton linac the beam arrives earlier than the crest to provide longitudinal focusing, means that phases and amplitudes are more sensitive to cavity resonant frequency variations than is the case for normal-conducting cavities. The two issues of concern are the Lorentz-force detuning, and microphonics. Cavity stiffening methods and beamloading compensation can generally be employed to mitigate these effects. 
R\&D issues focus on development of accelerating structures for the intermediatevelocity regime, higher accelerating gradients, and control of Lorentz force detuning effects and microphonics. LANL is actively pursuing the R\&D on spoke cavities. The spoke cavity work started at ANL in the early nineties. Recently, a 2-gap $\beta=0.175$ ) spoke cavity built at $A N L$ was tested at LANL. The measured values $\left(\mathrm{E}_{\mathrm{acc}}=10 \mathrm{MV} / \mathrm{m}\right.$ with $Q \approx 5 \times 10^{8}$ at $4^{\circ} \mathrm{K}$ ) are very promising towards the goal of a SRF structure for use at low $\beta$ region. The 2-MW, 1-GeV US Spallation Neutron Source (SNS), which is presently under construction, will be the first application of superconducting $R F$ technology to a proton linac. It uses a SRF structure for the high velocity regime; approximately $80 \%$ of the energy gain is from the superconducting linac sections.

One of the appealing advantages of a linac built entirely with SRF cavities is the potential flexibility during operation. Broader velocity acceptance means that in principle the linac can be re-tuned in the event of a klystron/cavity failure to deliver beam at the specified energy and current. However, needless to say that operation under such scenarios has to be supported by the built-in design specifications. The choice between normal conducting and SRF linacs is intimately tied to the question of macro-pulse length of the beam. For CW beam, SRF is the obvious choice; however, for pulsed-beam operation as the beam macro-pulse length gets shorter, the cost savings from electric power over the operational life time of the machine has to be weighed against the relative capital costs.

\subsection{RF control:}

The linac high-power RF systems are critical in terms of cost $(\sim 1 / 3$ of the linac cost) and availability ( $60 \%$ of the LANSCE linac downtime comes from the ion sources and RF system). Actual experience (LANSCE linac, LEP, SLAC, as well as development work for SNS and TESLA) provides a solid base for performance. Improvements can benefit from an $R \& D$ program focused on cost reduction, reliability and performance upgrade.

\section{$\underline{\text { R\&D Plan }}$}

- Decide on the choice of RF frequency.

- Determine the choice of the RF-source unit power with respect to the phase and amplitude controls (number of cavities per RF source, use of high-power sources to reduce the cost). (for SRF only)

- Develop high-performance HV power supplies for long pulsed ( $>1 \mathrm{~ms}$ ) and CW operation (following the work done on IGBT based HV PS at SLAC, LANL and industry).

- Study RF system schemes with redundancies allowing high reliability and availability as well as limited beam interrupts in case of failure of a component (work being done at the SNS and APT). (for SRF only)

- Develop high-performance RF control systems (feedback and feed-forward, including piezo-electric fast feed-forward) for accurate phase and amplitude controls during normal operation, tuning phases or non-standard operation (missing cavity...). (for SRF only)

- Develop high efficiency, high duty cycle RF sources to reduce the total construction plus operation cost (IOT, multi-beam klystron). 


\subsection{HEBT and RTBT:}

High intensity transfer lines demand very low losses (of the order of $1 \mathrm{~W} / \mathrm{m}$ ) for hands-on maintenance. Important considerations include correction of linac energy and position jitter, beam painting in transverse and longitudinal phase space, and diagnostics and equipment protection, in particular from target radiation in the RTBT.

$\underline{\text { R\&D Plan }}$

- Improve collimator systems, which, unlike collimation in rings, are single-pass. Collimator efficiencies are generally not high (80-85\%). (Category A)

- Develop profile monitors to replace wire systems, which are likely to melt under the high intensities expected. Profile monitors are needed to measure halo of 5 to 6 orders of magnitude lower in intensity. (Category A)

- Perform experimental studies on the energy and phase jitters coming from the linac and determine the required corrections. (Category B)

- Consideration should be given to designing bending systems that are achromatic under space charge. Linear space charge codes already exist (RAL: KVBL, $\mathrm{SPACEX}$ ) and partially meet this requirement. (Category $\mathrm{C}$ )

\subsection{Space charge effects:}

One important topic of high-current proton linacs is to identify sources of beam loss that originate from space charge and the formation of halo. There are currently a number of projects/proposals worldwide for which detailed beam dynamics studies are under way: SNS, KEK/JAERI, CONCERT/ESS, CERN-SPL, Fermilab Proton Driver, BNL Proton Driver, RAL Proton Driver, and the AAA at the LANL. These projects/proposals have been presented at Snowmass. The LEDA-experiment of LANL aiming at a first experimental verification of the current understanding of halo formation has been discussed in this context. Simulation codes to respond to these tasks have been discussed in M6, and in a joint session with the Parallel Computing working group. Although work on existing projects is progressing there is still need for considerable effort to integrate conclusions from beam dynamics into the design and/or diagnostics concepts. Future (even more powerful) proton driver projects will benefit from this development.

Issues for Study

Space charge driven resonances are an intrinsic source of rms emittance exchange and growth independent of a particular lattice. They are controlled by the longitudinat totransverse tune ratio and the amount of non-equipartition in the high current bunches. Halo as the main source of beam loss requires some kind of envelope mismatch (in 3-D) and/or steering errors. The current understanding is that resonant interaction between core and tail particles drives a halo, which is a fully $3-\mathrm{D}$ process. Initial mismatch/steering, and the effect of random or correlated errors in quadrupole gradients and RF amplitudes/phases need to be studied systematically, and also correlated with space charge resonance. Such studies are currently evolving with the existing projects; in this context it is recognized that considerable work needs to be done to understand the mechanisms and determine the radii of halos at the level of $10^{-4}$ fractional intensity. 
$\underline{\text { R\&D Plan }}$

- Develop code to cope adequately with the 3-D space charge requirements, including error studies with space charge. This has the highest priority. Due to the lack of code validation with experiments in this field, comparison and benchmarking of 3-D space charge codes must be undertaken in parallel to raise the confidence in simulation codes as design tools for high power drivers. The needs of high space charge resolution and of the large error sets to be considered may require significantly increased use of massive parallel computation.

- Experiments in this field appear to be difficult. Due to the importance of verifying the physics concepts behind our present halo and loss modeling, and the need to check the adequacy of diagnostics concepts, more efforts should go into experiments. One shouldn't wait until the commissioning of one or several of the current projects. The experience gained with well-equipped experimental verification, as well as simulation studies, will be useful for commissioning.

- Diagnostics methods for halos at the $10^{-4}$ level exist, but more R\&D is needed to make these methods applicable to proton driver linacs. Also, R\&D is needed to make emittance measurements (at various levels of intensity) feasible in the space charge dominated regime. In an experiment similar to LEDA such diagnostics would be placed at the necessary positions along the channel, which is also equipped with RF bunchers.

\subsection{Diagnostics:}

The acceleration and transport of high power beams present new challenges for beam diagnostic systems. Conventional measurements will continue to be required, but not all traditional methods are acceptable in the presence of high power beams. Operating conditions may need to be modified to permit the use of traditional instruments. New measurements will be required to detect, diagnose, and prevent small fractional beam losses that can damage accelerator components and produce unacceptable levels of residual radiation in high power machines. Monitors that can directly measure beam halo must be developed because the performance of new high power machines may well be halo dominated. If the new machines are to operate as expected at as yet unachieved performance levels, the diagnostics must keep pace. The working group was reminded, "If you keep doing what you've been doing, you will keep getting what you've got."

Devices that can produce credible profile measurements of high power and high space charge beams are critical to beam emittance and other transverse parameter measurements. Beam mismatch that couples to space charge distribution oscillations have been determined to be a major factor in beam halo development. Traditional multiwire or scanning wires are time-proven devices for profile measurements, but they exhibit severe shortcomings for application to high power beams and in superconducting linacs. $\mathrm{BNL}$, as part of the SNS project, is currently researching "laser wire" techniques as a solution to this problem for $\mathrm{H}^{-}$beams. Good progress has been made and the technique appears to be an attractive potential solution though measurement of high-energy beams with suitable resolution has yet to be demonstrated. We strongly encourage that work to be continued. At the same time, R\&D into other innovative solutions to this very 
important problem should not be neglected. Ion profile monitors and fluorescence-based monitors are options that deserve continued development, although high space charge beams present particular difficulties to these methods. Full transverse emittance measurements are most important and perhaps only obtainable at either end of a long linac structure. With suitable beamline design, laser-based extraction of short pulses may be used for emittance measurements without interrupting normal operation. This is an example where beamline designs may need to include specific considerations for particular measurements. Problems using wire harps immediately upstream of targets or beam dumps due to back scattering were noted.

Diagnostic systems with sufficient bandwidth to observe beam parameter variations during the pulse will be especially important for long pulse linacs. With chopped linac beams, multi- $\mathrm{MHz}$ bandwidth may be necessary to observe the transients due to chopping.

The trend toward superconducting hadron linacs will have a major impact on beam instrumentation. There are serious concerns related to contamination of the superconducting cavity surfaces during equipment installation and operation. Moving parts in traditional instrumentation like wire scanners, harps, and emittance monitors pose the threat of liberating dust, flakes, or other particulates that can migrate into the cavities. Intercepting devices with the potential for breakage, ablation or sputtering of material heated by the beam also risk contamination. All devices to be installed in the vicinity of superconducting RF, even non-intercepting and non-moving devices, will be subjected to stringent cleansing requirements prior to installation. SNS will be at the forefront of this new challenge for hadron machines.

Longitudinal measurements of linac beams will become more important as demands for enhanced performance are to be met. On-line energy measurements and energy spread measurements will be important to SNS beam transport and ring injection commissioning and operation. Precision beam phase measurement may permit time-of-flight energy measurement methods to be used. It is quite possible that the shape resonance bump in the cross section near the $2 p$ threshold can be used for $\mathrm{H}^{-}$beam energy spread measurements. The laser-excited $\mathrm{H}^{0 *}$ shape resonance can also be used for absolute beam energy measurements, to complement time of flight or beam rigidity measurements. Beam energy jitter, which can be measured in a high-dispersion point in an arc, is a more important measurement than absolute energy. Thin halo scraper foils at a high dispersion point can measure momentum halos.

Development of an on-line, non-invasive bunch length/shape measurement would be valuable. Some form of a pulsed-mode-locked laser may be useful for bunch length measurement, but the issue of $\mathrm{H}^{0}$ background from residual gas stripping must be considered. The shape resonance (see above) may be useful. One approach was demonstrated at the LANL LINDA experiment.

\section{$\underline{\text { R\&D Plan (all in Category A) }}$}

- Develop non-invasive beam profile measurements and accurate on-line beam energy and energy-spread measurements.

- Develop specific beam halo monitors with compatibility with superconducting RF environments.

- Develop longitudinal bunch shape monitors having $\sim 10$ ps time resolution. 


\section{Ring}

\subsection{Lattice, aperture and corrections:}

The choice of lattice for the high intensity proton rings currently proposed has generally been between FODO, FODO with insertions, and doublet/triplet focusing structures. The relative merits depend on the requirements of the machine being designed. The relative simplicity of a FODO structure may often be sufficient, while a demand for achromatic arcs and long straight sections may suggest the flexibility of a structure based on triplets.

The lattice should include sufficient space for the four functions of injection, collimation, RF and extraction, and lattice parameters have to be such that each function can be satisfactorily achieved. The SNS approach has been to use 4-fold symmetry to put each operation into separate straight sections. JHF, Fermilab Proton Driver (FPD) and the LANL AHF synchrotrons adopt 3-fold symmetry with injection and collimation combined in one insertion Studies for ESS and the RAL proton drivers achieve decoupling and retain 3-fold symmetry by injecting in a dispersion region in a low field dipole in one of the arcs. Collimation to remove any momentum tail is then immediately after injection, where the dispersion is high. Detailed studies indicate that collimation efficiency could be higher with a doublet lattice than with a FODO structure.

With synchrotrons, an important issue is to avoid crossing transition during acceleration. The ring may be designed so that the machine's top energy is below transition, but in cases where this proves unrealistic, the problem may be avoided with an imaginary $\gamma_{t}$. This option has been chosen for the Fermilab Proton Driver and is one of two possible designs for the AHF. A "missing magnet" structure may also be used for dispersion modulation to avoid crossing $\gamma_{\mathrm{t}}$. However there is a drawback in that a larger number of quadrupole families are generally required in these "flexible momentum compaction" lattices. (This problem has been avoided in the AHF transitionless lattice, which has only two families of quadrupoles.)

With a few exceptions, chromaticity correction is necessary for increased dynamic aperture in a ring. Achievement of suitable phase advances for effective correction schemes puts demands on the structure of the lattice. $270^{\circ}$ per periodic section in both transverse directions is the most appropriate choice, although there are claims that flexibility in the vertical plane has some advantages. Choice of operating tunes is determined by the avoidance of resonances, the level of space charge in the ring, and with due regard to magnet errors in the machine. Resonance corrections (up to fourth order) need to be addressed including the avoidance of resonances that may cause emittance increase under non-linear space charge. Some resonance lines are more deleterious to performance than others. Computational tools already exist for quantitative examination of these effects. A design that allows the tune to be varied over a wide range is also desirable.

An additional demand made of proton drivers for neutrino factories or muon colliders is the need to produce high intensity short bunches of 1 to $3 \mathrm{~ns}$ rms duration. Such a requirement is imposed at a target to produce intense short bursts of pions and muons. The RAL proton driver designs based on synchrotrons use convergence in $\gamma_{t}$ to achieve the compression with reasonably low voltages $(\sim 500 \mathrm{kV})$. In these models, the momentum-dependence of transition energy needs to be carefully examined and 
correction schemes of non-linear effects devised using sextupoles and possibly higherorder multipole magnets. There is room here for R\&D (category $C$ ). The driver for the CERN neutrino factory study avoids the problem by working well below transition and using a separate compressor ring at high voltage $(\sim 2 \mathrm{MV})$ to create short bunches through non-adiabatic phase rotation. A specific lattice design for such compressor rings could form a subject for study, and might include an FFAG or a ring with superconducting magnets.

Many tried and tested computer codes exist for lattice design, including MAD, SYNCH, SAD, MAGIC, TRANSPORT and the relatively recent Lie algebraic tools MARYLIE and COSY- $\infty$. Apart from a new version of MAD, these take no account of space charge. However, space charge distorts the optics of a lattice and next-generation proton drivers could well rely on the use of linear space charge codes for more realistic integrated lattice design. Such codes exist at RAL (KVBL and SPACEX) and at CERN (AGILE) and have been extensively used in recent years in the design of machines such as ESS.

\subsection{Injection and extraction:}

Achieving the intensities required by the next generation of proton drivers demands nonLiouvillean techniques for injection into the ring. The two methods under consideration involve the conventional technique of $\mathrm{H}$ charge exchange using stripping foils or the relatively recent idea of stripping via an intense laser and optical resonator system. The latter has been promoted by JAERI/KEK as generating less particle loss and giving better control of emittance. To study this a collaborative study was set up between JAERI, LANL and RAL. Problems were encountered over the design of the undulators (which require a rapidly rising and falling magnetic field of about $1 \mathrm{~T}$ ) and with emittance increase. Since JHF does not foresee use of this technique, the study is largely in abeyance. However, the limit of foil stripping capabilities is being approached and higher intensities will demand alternative methods. In this respect, $\mathrm{R} \& \mathrm{D}$ of laser stripping injection is important and should be pursued as circumstances permit (Category $\mathrm{C}$ ).

$\mathrm{H}^{-}$injection via stripping foils is a tried-and-tested technique and there is much expertise in its use, for example at the Fermilab Booster, RAL ISIS and LANL PSR. Future proton drivers, whether used for generating spallation neutrons or in neutrino factories, will aim for active maintenance in the rings. The problems to be faced include uncontrolled beam loss caused by scattering in the foil, the removal of unstripped $\mathrm{H}$ and $\mathrm{H}^{0}$ and of the stripped electrons, and protection of the foil against excessive temperature rises. Careful choice of injection energy and bending fields (as in the ESS) can help reduce the $\mathrm{H}$ and $\mathrm{H}^{0}$ problem, but the injection region has nevertheless to be carefully designed to ensure that unwanted particles are safely transported to beam dumps. Excessive heating of the foil can be the result of traversals by re-circulating protons and much effort has been expended in devising painting schemes to reduce hits to an average below 3 to 4 per particle. Codes with varying degrees of sophistication have been developed at BNL, RAL, ORNL and FNAL to predict the temperature rises, and some bench marking and comparison with experiment would now be beneficial. As a rule of thumb, for carbon foils, the temperatures should not exceed $2000^{\circ} \mathrm{K}$. Other approaches to controlling temperatures include using larger rings to reduce the number of injection 
turns, or injecting into two rings to create bunches which are then combined at the target (e.g. ESS). With fewer particles per ring, the latter has the additional advantage of reducing space charge effects during subsequent storage or acceleration.

Space charge effects can also be reduced by painting a transverse distribution as uniform as possible during injection. (The preferred distribution at the target is more likely to be elliptical, but some re-distribution may be possible in subsequent RTBT transfer lines.) The various painting methods under study include the use of correlated and anti-correlated horizontal and/or vertical orbit bumps (e.g. SNS), vertical orbit bumps with dispersion painting (e.g. ESS, AGS, RAL and CERN proton drivers), and a combination of orbit bumps with directional variation of the incoming beam (FNAL Proton Driver). ESS also uses RF steering to assist painting. Different schemes create differently shaped beams (nominally rectangular, diamond, elliptical when space charge is ignored) and care needs to be taken to avoid those, which require large aperture. Intermediate control of the programmable bumps gives a range of particle densities. In synchrotrons where the injection period can cover decelerating and/or accelerating buckets attention needs to be given to RF voltages to ensure all particles injected are trapped.

The choice of ring tunes has emerged as a critical issue in avoiding emittance blow up. During the injection process, beams are highly non-linear and exhibit progressively increasing tune spread. Simulation studies have underlined that fourth-order resonances such as $2 \mathrm{Q}_{\mathrm{h}}-2 \mathrm{Q}_{\mathrm{V}}=0$ are to be avoided. Repeated modeling over a range of tunes can indicate the optimal choice.

It is generally agreed that injection-modeling codes are well benchmarked and adequate. Some future development may, however, be possible. Apart from work on laser stripping, the main aspect of an R\&D program related to injection studies should be work on improved stripping foils, structure, materials and lifetime.

Fast extraction is envisioned for many of the proton drivers, with the injection and trapping process designed to create gaps between bunches sufficient for the rise-times of the extraction kickers. The ESS kickers, for example, have a rise time less than $190 \mathrm{~ns}$ and give a deflection angle of $16 \mathrm{mrad}$. Slow extraction is also under consideration and with such systems the possibility of beam loss is more of a concern. An R\&D program needs to be devised to evaluate the feasibility of slow extraction schemes with beam loss below $1 \%$, and this can be tied in with a Fermilab-KEK beam experiment currently planned on the Main Injector at Fermilab.

\subsection{Space charge and halo:}

It isn't possible to separate space charge from other beam dynamics issues in high intensity rings, as many effects interact to influence each other. In the following, we describe areas in which space charge forces require strong consideration. In contrast to linacs, where longitudinal and transverse bunch sizes are comparable, longitudinal bunch sizes in rings exceed transverse bunch sizes. Accordingly, transverse tunes are orders of magnitude larger than longitudinal tunes, and considerations of longitudinal and transverse space charge can often be separated.

Longitudinal space charge effects must be considered as part of a complete longitudinal dynamics picture. These effects tend to spread the bunch and, if this 
spreading causes leakage from the bucket, they may require compensation, either through enhanced RF focusing or introduction of an appropriate impedance into the ring. Proper control of the longitudinal bunch is important in maintaining clean gap regions and also in avoiding excessive peaking of the longitudinal density, which could lead to harmful transverse effects.

In linear accelerators, beam mismatch is known to be a major cause of rapid emittance growth and halo. In high intensity rings, where space charge forces are much smaller than in linacs, this process is slower. During accumulation, which may require many turns, the time scale for the change in rms beam parameters due to injection is comparable to or faster than that for halo generation due to mismatch. Even so, it is important to simulate potential injection schemes in detail to assure that mismatch during painting does not lead to unwanted emittance growth or halo. This also applies to beam that is stored or accelerated subsequent to injection. The excitation of space charge or lattice resonance could speed up and enhance this process.

Space charge forces shift and spread individual particle (incoherent) tunes. However, it is not these, but rather the coherent tune of the beam, associated with its collective oscillations, which determines its resonant behavior. It is necessary to maintain the coherent tunes of the beam away from bw order (integer and half integer) and structure resonance. Failure to do so could result in significant beam broadening and emittance growth. It is essential to consider the separation between the horizontal and vertical coherent tunes because small separation can lead to coupling resonances that can lead to considerable emittance exchange. It is also possible that separation of the horizontal and vertical tunes by an integer value, particularly if the integer is a multiple of the lattice superperiodicity, or lattice-induced coupling will lead to emittance exchange.

The effect of space charge on lattice resonances in high intensity rings requires study. Working points are often selected to avoid lattice resonances, but such working points may lie sufficiently close to integer or half integer values that these resonances will be excited at high intensity due to coherent tune depression. Choice of working points to avoid this situation often places the high intensity tune distribution across one or more higher order lattice resonances. The effect and possible correction of these resonances for high intensity beams requires study.

The effect of longitudinal bunching on transverse space charge behavior can be significant in that the local (longitudinally) current density can greatly exceed the average for the bunch. Careful attention should be paid to the longitudinal injection, painting, and dynamics to avoid excessive bunching factors.

The compensation of space charge forces by electron beams is also an interesting possibility, and studies are being conducted at KEK and Fermilab.

The effect of space charge on impedance driven instabilities needs to be studied. For transverse impedance, this requires a three dimensional description of the space charge.

A complete description of the electron cloud instability will ultimately require incorporation of the beam response, and this will require a three dimensional description of the space charge.

$\underline{\text { R\&D Plan }}$

- The comparison of detailed space charge simulations with experimental results should be performed for rings such as PSR, AGS Booster, Fermilab Booster, and 
CIS. Such comparisons are already underway at PSR and CIS and a commitment has been made to begin studies at Fermilab.

- Diagnostics for the measurement of both dipole and quadrupole moments should be implemented in high intensity rings and in simulation codes.

- Studies of the behavior of high intensity beams in the presence of space charge and lattice resonance should be carried out. The correction of the lattice resonance should also be studied.

- The extension of high intensity ring beam space charge codes to include new physics models such as impedance, nonlinear lattice (single particle) transport terms, and self-consistent electron cloud dynamics, should be carried out. These models should be benchmarked with each other, with theory, and with experiment if possible. The application of such models to real problems will become computationally expensive and will necessitate the use of high performance parallel computing techniques and facilities.

\subsection{Beam stability and impedance:}

Most of the accelerators built so far are designed with sufficient or even largely overestimated margins to avoid or to cope with collective instabilities. This may become impossible in the design of the next generation proton sources like the compressor ring for a neutrino factory or a muon collider, which are expected to operate in the regime of kilo-Amperes peak beam current at low to me dium energy. In these machines, the margin for errors could be small and the beam intensity will be pushed close to or even beyond the stability limits. Accelerators of this class will be costly and so could be any over design or retrofit. We must have an improved understanding of instabilities, better estimates of instability thresholds, carefully planned impedance budget, and good preparations for coping with instabilities.

It is known that the observed longitudinal instability threshold may disagree with that estimated from the Keil-Schnell criterion. Examples are the ISIS synchrotron and the CERN PS. Though better agreement may be achieved by applying the stability thresholds derived from assumed simple equilibrium beam phase spaces, there is still no guidance on the accuracy of extrapolating this kind of criteria to the regime of severe potential-well distortion expected in the next generation high-intensity proton accelerator. In transverse beam dynamics, a similar problem also emerges in applying the existing theories. The ef fect of sudden large space-charge tune shift that occurs during bunch compression is still not clear. One of the techniques proposed for bunch compression is to operate near transition. The usual formula for estimating the stability threshold has doubtful validity in this regime. Thus, better understanding of the instabilities and improved approaches for estimating stability thresholds need to be established through more analytical studies, computer simulations, and possibly with beam experiments.

A recent development is the availability of large computing power using parallel processing and fast CPU. This should be exploited for investigating cases of combined effects, which are difficult or impossible to handle analytically. Examples are cases with large transverse impedance combined with space charge and/or electron cloud, synchrobetatron coupling, betatron resonance, etc. As well, and in parallel, it is desirable that the simulations be validated by results from beam experiments. As yet not fully explained 
effects such as the fast vertical instability at the PSR and the slow high-energy losses in the FNAL Booster can be tackled in this way. Though such work is already ongoing at many labs (FNAL, ORNL SNS, CERN, BNL), a more collaborative effort is required.

In connection with the instability studies, it is necessary to ameliorate impedance modeling and measurement. Here as well, computations and simulations using real boundary conditions can help the theoretical development. The customary single-pole broadband impedance model should be examined for its applicability and for possible modifications. Also, it is desirable to check whether localized sources of beam coupling impedance can be adequately represented by a single impedance function which is assumed to be distributed around the ring in analytical studies but highly localized in some numerical simulations using the approximation of one or a few kicks per turn. Impedance measurements based on coherent tune shifts versus beam intensity and instability growth rates versus chromatically require a proper analysis, including the effect of coherent and incoherent tune shifts for vacuum chambers of flattop and flat bottom shape.

Studies of a few special devices having high potential like inductive inserts and special beam pipes have to be actively pursued. Preliminary experimental data from LANL and KEK have shown encouraging results using inductive inserts to compensate the longitudinal space-charge force. Practical use of this kind of device is being seriously considered. For example, the proposed CERN accumulator requires an inductive $\mathrm{Z} / \mathrm{n}$ of $70 \mathrm{Ohms}$, with a real part no larger than $1 \mathrm{Ohm}$ up to the beam pipe cutoff. R\&D needs to be done. The coupling impedance of thin resistive layers, such as metallic coatings of ceramic vacuum chambers in kicker magnets, requires further investigations. Theoretical studies have shown that the beam image current flows in the resistive layers even in the low-frequency regime, when the skin depth ä is larger than the layer's thickness, unless external structures offer alternative paths of lower impedance. At very low frequencies, where $a^{2}$ exceeds the product of the chamber's radius times the layer's thickness, one may expect a significant reduction of the real part of the transverse impedance. These predictions have been validated by preliminary bench measurements (wire method) at CERN and tests with beams at the EPA. More impedance measurements with more realistic (kicker) set-ups are recommended.

Attention should also be paid to the development of new techniques in feedback control for coping with instabilities. For example, progress made in the active damping of instabilities in some electron machines deserves serious consideration.

$\underline{\text { R\&D Plan }}$

- Establish approaches for improved estimates of thresholds of fast instabilities, both transverse and longitudinal. Collaborative effort is needed for resolving poorly understood instabilities in existing machines.

- Place currently used models such as the broadband resonator and distributed impedance on a firmer theoretical basis.

- Actively pursue development of inductive inserts that have large inductive impedance and very small resistive impedance.

- Carry out impedance measurements and analysis based on coherent tune shifts $v s$. beam intensity, and instability growth rate $v s$. chromaticity, including that for vacuum chambers of flattop and flat bottom shape. 
- Develop new technology in feedback implementation.

\subsection{Electron cloud:}

Electron cloud effects are increasingly recognized as important, but incompletely understood dynamical phenomena. They can severely limit the performance of the next generation of high-intensity proton rings such as LHC, SNS, and the Proton Driver. Deleterious effects include two-stream instabilities (e-p), emittance growth, increases in vacuum pressure, added heat load at the vacuum chamber walls and interference with certain beam diagnostics. Extrapolation of present experience to significantly higher intensities is highly uncertain given the present level of understanding. A comprehensive R\&D program including experiments, theory and simulations is clearly needed to better understand the phenomena, pin down essential parameters and develop proven remedies.

At this time, significant electron cloud effects have been observed at both lepton and proton machines, such as the KEK-B, PEP II, BEPC, the CERN PS, SPS, the LANL PSR, etc. Among the proton machines, a strong, fast transverse instability long observed at the Los Alamos PSR is almost certainly e-p. Copious production of electrons by a type of beam-induced secondary emission avalanche (aptly referred to as "trailing edge multipactor") has been observed there and is strongly suspected as the dominant source of electrons driving the instability. The coasting beam instability observed at the AGS Booster is also thought to be e-p. Evidence for significant electron cloud production by beam-induced multipactoring has been observed at the CERN PS and SPS when configured for LHC injection parameters. The electron cloud buildup depends critically on the intensity, spacing and length of the proton buckets, as well as on the secondary electron yield (SEY) of the beam pipe surfaces (especially at very low electron energies of a few eV). The added heat load on cryogenic systems, emittance growth, and instabilities are the main concerns for LHC.

Simulations of the electron cloud production in proton rings using codes such as POSINST, ECE and ECLOUD have had some notable successes in modeling many aspects of this phenomenon, including single- and multi-bunch instabilities for rings with short bunches. However, the simulations are limited by uncertainties in key parameters describing the interactions of low energy electrons $(<20 \mathrm{eV})$ with accelerator surfaces. Of particular iniportance is the SEY, including reflected or re-diffused electrons for low energy incident electrons, which are very difficult to measure directly. A high value, $\sim 0.5$, is needed to reproduce some of the features of the electron cloud buildup at the PSR. Methods to directly measure the SEY and the energy spectrum from low energy incident electrons $(<20 \mathrm{eV})$ for the surfaces being considered are sorely needed and should be a high priority R\&D activity along with studies of the important dependencies of these parameters on beam scrubbing (conditioning effect) and other surface treatments.

Accurate theories and models of the e-p instability dynamics for bunched beams are essential for predicting the performance of future rings. Rigid beam, centroid models for coupled electron and proton motion for coasting beams have provided valuable insights but appear to be too simplified and contain too many free parameters for reliable extrapolation to the next generation rings. These models have provided reasonable estimates of the unstable dipole modes and their scaling with intensity. They have produced plausible predictions for instability threshold intensities, given the uncertainties 
on parameters such as average neutralization. However, estimates of growth rates and behavior beyond threshold are in rather poor agreement with observations.

Some extensions of centroid models to bunched beams have been undertaken but more work is needed. It is essential to develop better insights into how the observables (mode structure, thresholds, growth rates and behavior above threshold) are changed for bunched beams. Ultimately it will be desirable to include electron generation in the dynamical model for the instability.

Progress has been made (at PPPL) in developing fully kinetic simulations based on self-consistent solutions of the Maxwell-Vlasov equations for coasting beams in a smooth focusing approximation. Threshold estimates and growth rates from these computations show reasonable agreement with observations. However, the computational power needed even for this simplified case is immense. Extension to bunched beams in a strong focusing ring lattice presents a formidable computational challenge. For a complete model it will be necessary to incorporate the physics of electron cloud generation At this time it is difficult to estimate the effort needed to carry out such program to completion. Nevertheless a way should be found to continue an ongoing effort to develop models based on this approach.

Most issues must be settled by a combination of theory and experiment. Much of what is known of electron cloud effects was first encountered in experiments. Retarding field analyzers (RFA) developed at ANL permit observation of the electrons striking the wall. Much has been learned from these data. However, the electron density in the beam is of fundamental importance to instability dynamics and is not directly measured by the RFA. Development of methods for its direct measure is a high priority goal. Such data would provide definitive tests of models for both electron generation and instability dynamics. Of equal importance would be measurements of the impedance produced by the electron cloud. Beam transfer function methods come to mind but are challenging to implement in accumulator rings such as PSR and SNS where the beams are stored only for a short time (milliseconds) before extraction.

Successful prevention, mitigation or cures for the undesirable effects of the electron cloud are the ultimate goal for the new machines. Definitive tests and demonstrations of potential cures should be part of the long term R\&D program. They fall into two broad categories: (1) measures to suppress generation of the electron cloud such as TiN coatings or other surface treatments, clearing fields, antechambers, weak solenoid fields (in field-free regions), and beam scrubbing plus (2) mechanisms for increased damping of the two-stream. instability such as measures to increase Landau damping or feedback systems for active damping. It may be necessary to use a combination of methods to reach the performance goals set for the new machines.

Weak solenoids (in field free regions), use of materials with low SEY, and beam scrubbing, accompanied by a strong reduction of SEY after an accumulated electron dose of a few $\mathrm{mC} / \mathrm{mm}^{2}$, are very promising cures against the electron cloud build-up. Use of materials with low SEY or coating of the vacuum surfaces with materials such as TiN, which lower the SEY, would help to reduce the cloud generation from mechanisms such as beam-induced multipactoring where secondary emission at the walls plays an important role. TiN coating of a straight section at the LANL PSR showed a dramatic suppression of the electron signal striking the wall. It would be important to determine if this resulted in a reduction of the electron density at the beam. Since copious numbers of 
electrons are observed at all location including dipoles and quadrupoles, the definitive test of TiN would be to coat the entire PSR ring and measure its effect on the observables of the e-p instability. This would entail an expensive time-consuming retrofit of a fairly radioactive ring and is not currently planned. A case could be made that the results of such an experiment are of sufficient importance to the new machines that it be made a DOE priority and funded as an $R \& D$ activity.

$\underline{\text { R\&D Plan }}$

- Develop and exploit improved codes to simulate generation of the electron cloud especially for long bunch machines such as PSR and SNS. A companion effort should include measurements of the SEY from low energy electrons $(20 \mathrm{eV})$ and various accelerator surfaces.

- Develop adequate theories, models and simulations for the dynamics of the e-p instability. The goal is to develop models and theories that could be used with confidence to predict the performance of the new machines. This implies theories and models that have been adequately tested and verified by experiments.

- Develop diagnostics and carry out experiments designed to thoroughly characterize the electron cloud and the impedance it presents to the beam. This should include methods to directly measure the electron density in the beam.

- Develop experiments and diagnostics to fully characterize the threshold intensities, mode structure, instability growth rates, and emittance growth for the e-p instability. These measurements and data on the electron cloud can be used to test dynamical models for the instability.

- Develop and test potential cures for the electron cloud effects including the e-p instability. A definitive test of TiN as a cure for the e-p instability at the PSR would resolve an important technical risk for the new proton machines, in particular for the SNS. The feasibility of active damping of the ep instability should be carefully examined, as it may also be effective and needed, especially for the long-bunch machines such as SNS.

\subsection{Beam loss, collimation and protection:}

The collimation system and shielding designs presented at Snowmass are based on realistic simulations using different Monte Carlo codes: GEANT, FLUKA, MARS, STRUCT, K2, ORBIT and others. Regulatory requirements for external shielding, handson maintenance and ground water activation are taken as the limits to be met. A common strategy adopted in all projects is based on beam loss localization in a specially designed section (met to match the collimation system requirements), thus reducing irradiation of the rest of the machine to acceptable levels. As all long straight sections of the machine must be equivalent for superperiodicity preservation, the collimation system has to be located in a zero dispersion straight section, which is optimized also for injection, RF and extraction. Additional collimators are necessary in low energy high intensity machines compared to the traditional 2-stage collimation approach.

Several different concepts for off-momentum particle collimation were presented: 
- Location of the primary and first secondary off-momentum collimators in a high dispersion region in the arc upstream of the collimation section (Fermilab Proton Driver).

- Use of a very thin (1 mm graphite) primary collimator in a high dispersion region of $\operatorname{arc}(\mathrm{JHF})$.

- Use of a kicker-magnet pulse between bunches for off-momentum particles deflecting them to the collimator located in a zero dispersion region (SNS).

- ISIS off-momentum collimation is done in the straight section immediately after the injection dipole, where the dispersion is fairly high.

Additional simulations should be done using different codes to validate the chosen collimation system design for a specific machine. Sensitivity analysis of collimation efficiency with respect to machine parameters stability (tune variation, orbit deviation, secondary collimators offset with respect to the primary ones, etc.) is very important.

$\underline{\text { R\&DD Plan }}$

- Validate and benchmark code (STRUCT, K2, ORBIT):

- code vs. code

- code $v s$. theory

- code vs. experiment

- Engineer and perform experiments on the collimator and beam dump designs with respect to material, cooling, impedance and reliability and cost reduction.

- Perform simulations and experiments on the bent crystal collimation in high and low energy machines (RHIC, Protvino U-70).

- Perform simulations and experiments on the use of betatron resonance for halo collimation (Fermilab $8 \mathrm{GeV}$ Booster).

\subsection{Magnets and kickers:}

The machines discussed in the M6 working group fall under three broad categories:

i Rapid cycling synchrotron (possibly cascaded)

- High energy linac + accumulation ring

" Fixed Field Alternating Gradient synchrotron (FFAG)

From the standpoint of magnet technology, each one of these technologies presents specific requirements and challenges. In rapid cycling synchrotrons, space charge effects are mitigated by minimizing the circumference and by spreading out the bunches, both transversely and longitudinally. Small circumference favors higher magnetic field; large transverse beam dimensions imply large physical aperture. Finally, since beam power is the product of bunch population, energy and cycling frequency, there is obviously a compromise between these three quantities. In general, the frequency is limited to approximately $50 \mathrm{~Hz}$ because of eddy current effects.

\section{Rapid Cycling Synchrotron}

In the Fermilab Proton Driver study, the field is set at $1.5 \mathrm{~T}$, the physical magnet aperture is 5 in $\times 11$ in and the cycling frequency is $15 \mathrm{~Hz}$. Because of the large stored magnetic energy, the power supply is of the resonant type (with a 2 nd harmonic to reduce RF power requirements). The combination of high field and high frequency leads to 
unacceptable eddy current losses and current distributions unless special water-cooled stranded conductor is used. This conductor is available commercially; however, there is virtually no experience with its application in magnets. An R\&D program is needed to understand how to make good electric al and mechanical connections and how to design magnet ends in view of the limited bending radius of the conductor. The program should also cover voltage-to-ground electrical insulation. Operation at $1.5 \mathrm{~T}$ is also challenging because of the need to ensure good dipole/quadrupole tracking through the cycle (tune control). For the $\mathrm{PD}$, it has been designed that this tracking should be on the order of 0.001 . To meet this requirement, quadrupoles and dipole share a common bus and residual tracking error is compensated by a dynamic correction system.

\section{High Energy Linac + Accumulation Ring}

This approach is used by SNS and is under consideration for other machines such as ESS. Space charge is mitigated by injecting at high energy $(\sim 1 \mathrm{GeV})$ into a fixed energy accumulation ring. The magnets here are much more conventional and are certainly less challenging although radiation damage is an issue.

\section{$\underline{\text { FFAG }}$}

KEK has embarked into a ambitious program that will lead to a Neutrino Factory based on cascaded FFAG machines. A small-scale prototype machine has been built. FFAG machines present many advantages; in particular, they offer a very large dynamic aperture that is ideal for efficient capture. FFAG magnets are inherently 3-dimensional; in particular they are profiled to ensure that the tune remains constant during the entire acceleration cycle. This used to be a non-trivial and costly proposition. Advent of fast computers combined with the availability of reliable 3-D magnetostatic codes (e.g. TOSCA) has changed this state of affairs. R\&D is needed to understand how efficiently to optimize the magnet profile. For a high energy FFAG, superconducting magnets will be necessary. Some designs have been proposed; more work will be required to produce practical designs.

\section{$\underline{\text { Kickers }}$}

Kickers are an important element in all modern synchrotrons. Traditionally, thyratronbased modulators have been used. Stacked Mosfet modulators are under development bọth for the DARHT project and the proposed AHF project at Los Alamos. These

modulators offer both fast rise times and fall times $(10-20 \mathrm{~ns})$ and high voltage (20 - 50 $\mathrm{keV}$ to a $50 \mathrm{Ohm}$ load). Such modulators coupled with constant-impedance stripline kickers or lumped element kickers offer important performance advantages in many applications at a cost comparable to thyratron modulators.

\subsection{Power supplies:}

There are three magnet power systems in common use today: bridge rectifiers directly comnected to the power grid, bridge rectifiers with local energy storage in a motorgenerator (flywheel) set, and resonant power systems with local energy storage in capacitors and chokes. In choosing among the options, many factors must be considered. First, come the requirements of the users - flexibility of magnet current programs and 
accuracy of control of flattops and flat bottoms. Another factor is stiffness of the local power grid; sites at the end of long power lines must pay more attention to disturbances put back on the power lines and the likelihood of larger line fluctuations. An overriding consideration is cost, both capital and installation cost. There is no single solution that is optimum for all situations, and all factors must be analyzed for each proposed machine. As a general rule, the higher the repetition rate, the larger is the peak power (often mostly reactive) drawn from the grid, and the more likely is the need for local energy storage. Thus, most "rapid cycling synchrotrons" built to date have resonant power systems. However, in very low repetition rate systems, it may also be advantageous to provide local energy storage so as to reduce peak energy demand and minimize grid disturbances. The technology involved in energy storage is well known, and design of such systems needs little or no R\&D. However, a variation of the conventional resonant power supply, namely, a duatharmonic system, which is adopted by the Fermilab Proton Driver design, needs considerable R\&D due to lack of experience in operating such a system.

Moreover, the dynamic range of the power systems, and the ever-increasing need for more precise control of the magnet current waveform can lead to very difficult control problems, especially on the "injection front porch" needed in many situations. High frequency $\mathrm{AC}$ bridge rectifier systems, such as those based on choppers and IGBT (or IEGT) rectifiers, offers the promise of higher gain-bandwidth product, elimination of reactive loading of the grid, greater efficiency, and better control. These advantages have yet to be demonstrated in any existing large synchrotron, and R\&D in this area may lead to larger dynamic range, less costly multi-ring designs, and better performance/reliability by better control of injection parameters. Some very important initial work in this area has been done at KEK, where a 1-MW prototype IGBT power supply for a rapid-cycling synchrotron was built and tested. The $280 \mathrm{MeV}$ proton cancer therapy synchrotron at Tsukuba University has the converter and chopper type of magnet power supplies (both for bending magnets and quadrupoles) using IGBT rectifiers. It has been operating since last year. This system, operating at $\sim 20 \mathrm{kHz}$, has impressive performance specifications. Initial reports are very positive, and we eagerly await further reports of operating experience with this power supply system.

\subsection{RF:}

Lattice magnet capabilities for moderate energy proton synchrotrons (circumference of a few hundreds of meters) dictate the needs for RF systems capable of developing up to 50 $\mathrm{kV}$ per meter in the range 1-20 MHz. The geometry of RF cavities capable of developing such voltages indicates the need for magnetic energy storage material with saturation magnetization beyond the range of available ferrites. New crystalline soft metallic alloys (MA) Finemet $\circledast$ (Hitachi Co. Japan) and Metglass ${ }^{\circledR}$ (Vitrovac, Germany) appear to be well matched to the technical requirements of such accelerating cavities. The iQf product of such cavities is a function of the geometry of thin tape wound cores. The inductance and $\mathrm{Q}$ of a core may be adjusted to a particular frequency requirement by the introduction of radial reluctance gap cuts of varying width. As a part of the US-Japan HEP collaboration a prototype Finemet RF cavity has been built and tested at Fermilab. The cavity is presently installed and operates at $7.5 \mathrm{MHz}$ in bunch coalescing service. The voltage gradient and power consumption characteristics are consistent with design 
expectations. However, RF power consumption and cooling problems are inhibiting the development of large systems of RF cavities. An increase in the energy storage to dissipation ratio $(Q)$ of perhaps one order of magnitude would be a major factor in sinnplifying the design and construction of large systems.

In addition to the synchrotron accelerating cavities described above there is a development program directed at low frequency $(\sim 5 \mathrm{MHz})$ very high gradient $(\sim 1 \mathrm{MV} / \mathrm{m})$ burst mode RF cavities for proton bunch rotation and muon manipulation. The voltage hold-off properties of large cylinders of high strength ceramic (alumina, etc) require investigation in this context.

Furthermore, a requirement for burst-mode RF cavities in the $100-200 \mathrm{MHz}$ range, capable of rapid phase modulation of gradients of a few MV per meter has emerged in the context of muon acceleration in an FFAG lattice.

\subsection{Beam loading and compensation:}

For the currently envisioned generation of neutron and neutrino sources of approximately $1-2 \mathrm{GeV}$ (e.g. ISIS, SNS, ESS, SLP) the choice of full energy injection from a linac into a fixed energy compressor ring eliminates many of the beam loading problems associated with fast-cycling synchrotrons.

The present generation of $\mathrm{e}^{+} \mathrm{e}^{-}$colliders (asymmetric B-Factories) has done much to pioneer advanced beam loading compensation techniques, both active (PEP-II) and passive (KEK-B) measures that would have been used at SSC and that will surely be used at future very high energy machines (VLHC). The issue requiring R\&D is that of power handling.

An area where the matter is less clear is that of medium energy ( 10 to $20+\mathrm{GeV}$ ) proton synchrotrons with say $10^{14}$ protons per pulse, for production say of $1 \mathrm{GeV}$ neutrinos. High average intensity dictates rapid cycling which in turn demands high effective accelerating gradients. Presently available technology uses either low R/Q (ferrite-type) or high gradient (MA type), but not both. It is anticipated that development of the split-core MA cavity for the JHF synchrotrons will show some promise of filling the gap but beam loading at beam revolution harmonics and power density levels needs more work. Perpendicular bias ferrites with high $Q$ are also worthy of further investigation, though the gradients are lower.

There is a potential problem specific to proton driver type machines worthy of study. A cavity with large gap and tube capacitance and heavily inductively loaded with, say, Finemet behaves almost as a lumped element resonator. One may imagine the case that the resonance is sufficiently broadband to span several RF harmonics. At high current, say $10^{13}$ protons per bunch, the RF waveform becomes heavily distorted (modulations up to $70 \%$ ) at each passage of the bunch. The usual vector feedback of gap voltage is not of sufficient bandwidth to correct, and other means are required. One possibility is to have a second large power tube use a beam feed-forward of the bunch shape to inject pulses of current to partially cancel the bunch induced voltage, leaving the residual to be corrected by the feedback. 


\subsection{Diagnostics:}

Serious attention should be paid to diagnostics necessary during multiturn injection when beam signals are complex and dynamic. Separating information of the most recent injected turn from that of previous turns is very difficult. Residual linac bunch structure on the beam dies out after a few turns in the ring. Intentional beam modulation to "tag" specific parts of the beam may be used. The dynamic range of beam intensity can vary by three orders of magnitude during the injection/accumulation time. Separating injection mismatch from intentional painting from emittance blow-up is a difficult diagnostic. Beam size can, by design, vary by up to a factor of thirty during injection and accumulation.

E-p instabilities have been shown to be important in some high intensity proton accumulator/compressor rings. Research into instrumentation that can clearly diagnose this problem is important. The Los Alamos PSR group has led the way in this effort in recent years and has demonstrated one such electron diagnostic instrument that they have developed. Fourier-transform analysis of high-harmonic betatron sideband signals from a wideband BPM is another technique that may be applicable to e-p diagnostics.

Credible beam profile measurement in circulating hadron machines is not regularly (if ever) achieved. Profile and halo measurements are important for diagnosing emittance growth and other historically nuisance problems that will result in significant beam power loss in high power machines. Turn-by-turn profile measurements are important to see injection evolution and envelope resonance. Other, non-intercepting, transverse "quadrupole moment" monitors that are sufficiently sensitive to typical beam aspect ratios should be developed. IPMs with strong magnetic fields seem to hold some promise for fast, unambiguous profile measurements but may impact sensitive machine lattice parameters. Specific halo monitors should also be developed.

The large tune adjustment range in the SNS ring may have impacts on diagnostics and especially feedback systems that depend on betatron phase differences. It is important that these lattice design flexibilities are appropriately conveyed to and understood by the beam instrumentation and feedback engineers.

Compressor rings, like SNS or PSR, find measurement of the "beam in the gap" to be an important measurement since that beam will be lost at extraction and produce unacceptable radiation. Measurements at the level of $10^{-5}$ on the sub-microsecond time scale are sought. This is a problem unique to accumulator rings, and not rapid cycling synchrotron rings.

Fast, accurate on-line transverse tune measurement and beam transfer measurements are useful. Many techniques are known for these measurements, but incorporating them into easy-to-use, on-line systems have proven difficult.

All time and frequency domain diagnostic signals become considerably more complex to deal with in rapid cycling synchrotrons in the intermediate energy range due to the fast velocity change of the beam.

\section{$\underline{\text { R\&D Plan (all in Category A) }}$}

- Work is needed on the whole area of diagnosing beam parameters (injection matching, painting, possible emittance blow-up, incremental intensity, etc.) during multiturn injection. 
- Develop circulating beam profile monitors that will produce credible results over a significant dynamic range and with turn-by-turn speeds.

- Develop methods for fast and accurate non-invasive tune measurements.

\section{General Remarks}

The interaction between lattice/optics design and beam instrumentation crucial for machine commissioning, operation and development is important to be considered early in the design stage. This requires early and continued interaction between physicists and instrumentation designers through the time of machine commissioning. SNS has made considerable progress in this regard, especially in the HEBT beamline design. Future machines should take this into account and further the early design stage integration of machine/beamline design with beam diagnostics requirements.

Integration of diagnostics systems (hardware and software) into control systems with easy-to-use interfaces and unambiguous results is critical to making the diagnostics part of operational machines. The best diagnostic is the diagnostic that gets used! Diagnostics that require operation by an expert will get used only by that expert. Development of the integration of instrumentation into controls systems is an area that requires continued and intensified attention.

It is imperative to strive for instrumentation that is able to make beam parameter measurements at the diagnostic and predictive level as opposed to simply measuring end results of important beam processes.

\subsection{Inductive inserts:}

High intensity rapid cycling proton synchrotrons require substantial RF voltage to provide longitudinal bunch focusing, acceleration and delivery of energy to the proton beam. The proton bunch image aurrents passing along the vacuum chamber conducting walls generate electric fields that reduce and may totally cancel the RF generated fields necessary to keep the beam bunched longitudinally. It has long been proposed (ca. 1966, A. Sessler and V. Vaccaro) that the space charge focusing force reduction may be reduced or eliminated through the intentional introduction of specific amounts of inductance into the accelerator vacuum chamber. In 1997 this concept was tested experimentally by the introduction of appropriate values of inductance into the vacuum chamber of the KEK (Japan) $12 \mathrm{GeV}$ proton synchrotron and the Los Alamos National Laboratory $800 \mathrm{MeV}$ Proton Storage Ring (PSR). In the first experiment (KEK) the predicted beneficial effect on the RF focusing force was observed by measurement of the incoherent synchrotron oscillation frequency of the protons. In the LANL case, improvement in the bunching factor and RF system performance was observed. A slightly improved version of the ferrite inductor initially installed in the PSR is now installed and operated routinely. This installation has resulted in a large increase in the PSR beam delivery capability and substantial cost saving. These encouraging results imply that in certain circumstances, it may be advantageous to obtain beam developed longitudinal focusing through the introduction of inductance, that would be impractical or impossible by external RF means. The Fermilab Booster is also installing several ferrite inductor modules to study their effects on the beam. Continued development of magnetic 
material properties could very well result in improved performance and cost savings in proton facilities through passive space charge compensation with installed inductance.

\subsection{FFAG:}

After 38 years of neglect, FFAG Accelerators have reentered the accelerator scene with the construction of the Proof of Principle machine (POP) at KEK. The use of FFAG is contemplated for medical ion synchrotrons, phase rotation and acceleration of muon beams, from muon production energies of several hundred $\mathrm{MeV}$ to storage energies of hundreds of $\mathrm{GeV}$. Interest is in both the classical scaling structures (zero chromaticity over the full momentum aperture) and non-scaling versions that are of interest for highenergy recirculators with linacs.

Scaling FFAG accelerators can operate at high repetition rate with smaller numbers of particles in each bunch, reducing space charge effects. Several bunches can be accelerated at the same time, even with a single (wideband) cavity. Intermediate stacking can be used to optimize RF acceleration and to take advantage of the reduction of space charge effects as particle energy is increased. The beam duty cycle can be controlled from very short (single turn) to nearly $100 \%$ (slow extraction).

Technology changes which have caused this resurgence are (1) three dimensional magnetic field programs which can be used together with mesh dynamics programs iteratively to design successful magnet systems, and (2) successful development of large insulating cramic vacuum seals and amorphous iron magnetic materials to make large aperture wideband RF cavities. These areas need further R\&D for wider application.

Nonscaling FFAG accelerators are being studied for use as recirculators for accelerating high-energy muons. These are (1) almost scaling structures with reverse bends and (2) versions similar to a conventional alternating gradient structure. The goal is to make the structures isochronous, or to have a similar change in orbit length for each passage through the accelerating structure. A common problem is that the path length is a quadratic function of momentum (momentum compaction linear with momentum). Under these conditions several unusual conditions can occur. First, the phase oscillation frequency will be independent of momentum, and no phase damping will occur. Second, the phase oscillation frequency can be so high that the acceleration structures must be divided into many sectors so that the phase oscillations will not be chaotic. Although the quadratic orbit length term may in principle be canceled with sextupole fields, so far no solution has been found which does not cause serious reduction of dynamic aperture. These problems become less severe as the energies are increased. Aside from further lattice studies, R\&D is needed in transient phase shifts in high frequency RF structures to adapt to the path length problem in the recirculators.

$\underline{\text { R\&D Plan }}$

- Prototype a cavity to study the ceramic sealing problem and the low Q cavity. (2 person-years, $\$ 150 \mathrm{~K}$ without RF source)

- Verify and evaluate tracking code. (9 person-years)

- Study fast and slow extraction; design a C-type kicker. (1 person-year)

- Prototype a magnet. (2 person-years, $\$ 150 \mathrm{~K}$ hardware, $\$ 70 \mathrm{~K}$ software)

- Work on diagnostics. ( 0.5 person-year for design, 2 person-years for prototyping) 
- Carry out a superconducting hybrid magnet design study. (1 person-year)

\subsection{Induction synchrotron:}

\section{Concept of Induction Synchrotron}

A novel proton synchrotron employing induction cells instead of radio frequency cavities has been proposed by K. Takayama and J. Kishiro, "Induction Synchrotron" N.I.M. A451 (2000) pp. 304-317. Its major feature is barrier bucket acceleration where acceleration and longitudinal focusing are independently achieved. In this sense, the induction synchrotron can be called a separated function type synchrotron in the longitudinal direction. Acceleration is given by a long step-voltage and confinement is done by a pair of barrier voltages with opposite polarity. These required step-voltage pulses are independently generated at accelerating gaps in induction cells. The barrier bucket acceleration provides great freedom of beam handling in the longitudinal direction. For instance, it is quite easy (in principle) to generate a beam with a desired length and desired momentum spread by controlling the time-duration between barrier voltage pulses and their height. Barrier bucket acceleration allows an ultimate use of longitudinal phase space and is quite effective in substantially increasing the beam intensity in synchrotrons, without increasing the local line density. An improved bunching factor is obtainable by employing a technique of symmetric painting. Key devices to realize the novel synchrotron are a ferri/ferro-magnetic material loaded induction cell and a modulator being rapidly switched in synchronization with beam acceleration.

\section{Status of R\&D Work on the Induction Device and Modulator at KEK}

Since 1999, the PS division in KEK has been developing the induction device using nano-crystalline magnetic material such as Finemet and a modulator triggered by a fast switching device using FET elements or a Static Induction Thyristor (SIT), in a close collaboration with the Tokyo Institute of Technology and Industries. After careful coreloss measurements for various magnetic-core materials, a prototype has been assembled and successfully operated with an output voltage of $4 \mathrm{kV}$ and a pulse width of $400 \mathrm{~ns}$ at $100 \mathrm{kHz}$. Recently its operation at $800 \mathrm{kHz}$ was demonstrated. From the operation of the prototype device, important features such as switching performance and heat deposit have been learned. After that a second prototype using the SIT as a switching element, replacing 96 FETs used in the first prototype, was assembled and demonstrated its capability with an output voltage of $3 \mathrm{kV}$ and a pulse width of $200 \mathrm{~ns}$ at $200 \mathrm{kHz}$. The unit cell, which is currently under design, has the following specifications: an output voltage of $2.5 \mathrm{kV}$, a pulse width of $450 \mathrm{~ns}$, a maximum repetition rate of $800 \mathrm{kHz}$, a physical length of $0.1 \mathrm{~m}$, and a core loss of $3 \mathrm{~kW}$.

\section{Application to the KEK $12 \mathrm{GeV}$ PS and the $3 \mathrm{GeV} / 50 \mathrm{GeV}$ Rings of the JHF Project}

An application to the KEK $12 \mathrm{GeV}$ PS (devoted to the K2K experiment) has been considered as a major upgrade to increase the beam current by a factor of two. The plan consists of formation of a long bunch in a permanent magnet $500 \mathrm{MeV}$ Accumulator Ring (AR) and its acceleration in the $12 \mathrm{GeV}$ PS. In the AR, 12 bunches injected from the Booster are combined in a barrier bucket to form a long bunch of order of a few microseconds, which is called a super-bunch. For acceleration, a total induction voltage 
of $25 \mathrm{kV}$ must be generated at a maximum repetition rate of $860 \mathrm{kHz}$. Substantial increase in the beam intensity and shortening of the injection time-period allow one to increase the average beam intensity by a factor of two. A proof-of-principle experiment of super-bunch acceleration in the KEK-PS is expected by the end of 2002.

On the other hand, there is a great potential to apply this concept to the rapid cycling $3 \mathrm{GeV}$ synchrotron and the slow cycling $50 \mathrm{GeV}$ synchrotron of the JHF Project. According to a possible plan for the former ring, chopped micro-bunch trains delivered from the $H$ linac are injected into a barrier bucket and the bucket is uniformly painted, resulting in a bunching factor of 0.76 . A quickly accelerated bunch is injected into a trapping barrier bucket in the $50 \mathrm{GeV}$ Ring and the trapping bucket is moved toward the barrier bucket for stacking. At the edge of the stacking core the fresh bunch is released, then the timing trigger of the one-side barrier voltage pulse is delayed by the pulse width of the fresh bunch. Eventually the fresh bunch merges into the stacked bunch-core. The process is repeated until a super-bunch, available for acceleration in the $50 \mathrm{GeV}$ ring, is generated. This induction synchrotron scheme will allow us to obtain a two or three times higher beam intensity, compared to the design value based on RF technology.

\section{List of participants}

\begin{tabular}{|c|c|c|}
\hline Name & Institution & E-Mail \\
\hline Rick Baartman & TRIUMF/Canada & baartman@1in12.triumf.ca \\
\hline Roberto Cappi & CERN/Switzerland & roberto.cappi@cern.ch \\
\hline Weiren Chou (convener) & Fermilab/USA & chou@fnal.gov \\
\hline Pat Colestock & LANL/USA & colestock(a)lanl.gov \\
\hline Sasha Drozhdin & Fermilab/USA & drozhdin@fnal.gov \\
\hline Miguel Furman & LBL/USA & mafurman@1bl.gov \\
\hline John Galambos & ORNL/USA & jdg(a,ornl.gov \\
\hline Jim Griffin & Fermilab/USA & igriffin@,adcalc.fnal.gov \\
\hline Helmut Haseroth & CERN/Switzerland & helmut.haseroth@,cern.ch \\
\hline Ingo Hofmann & GSI/Germany & i.hofmann@gsi.de \\
\hline Jeff Holmes & ORNL/USA & jzh@ornl.gov \\
\hline Rolland Johnson & IIT/USA & roljohn@aol.com \\
\hline Peter Kasper & Fermilab/USA & $\overline{\text { kasper@fnal.gov }}$ \\
\hline Shane Koscielniak & TRIUMF/Canada & shane@triumf.ca \\
\hline Jean-Michel Lagniel & CEA/France & imlagniel@,cea.fr \\
\hline Ka-Ngo Leung & LBL/USA & knleung@,lbl.gov \\
\hline Bob Macek & LANL/USA & macek@lanl.gov \\
\hline Shinji Machida & KEK/Japan & shinji.machida@,kek.jp \\
\hline Ernie Malamud & Fermilab/USA & malamud@,fnal.gov \\
\hline Sig Martin & Juelich/Germany & s.martin@,fz-juelich.de \\
\hline Fred Mills & Fermilab/USA & fredmills@aol.com \\
\hline Nikolai Mokhov & Fermilab/USA & mokhov@,fnal.gov \\
\hline Surbrata Nath & LANL/USA & snath@lanl.gov \\
\hline
\end{tabular}


Filippo Neri

Francois Ostiguy

Ben Prichard

Chris Prior

Hong Qin

Deepak Raparia

Thomas Roser

Francesco Ruggiero

Rob Ryne

Peter Schwandt

Bob Shafer

Yoshito Shimosaki

Ken Takayama

Arch Thiessen

Pete Walstrom

Tai-Sen Wang

Tom Wangler

Bob Webber

Jie Wei (convener)

LANL/USA
Fermilab/USA
LANL/USA
RAL/England
PPPL/USA
BNL/USA
BNL/USA
CERN/Switzerland
LBL/USA
Indiana U./USA
LANL/USA
KEK/Japan
KEK/Japan
LANL/USA
LANL/USA
LANL/USA
LANL/USA
Fermilab/USA
BNL/USA

fneri@1anl.gov

ostiguy@fnal.gov

prichard@1anl.gov

c.t.prior@rl.ac.uk

hongqin@pppl.gov

raparia@bnl.gov

roser@bnl.gov

francesco.ruggiero@(cern.ch

ryne@,lanl.gov

schwandt@iucf.indiana.edu

rshafer@1anl.gov

shimo@www-accps.kek.jp

takayama@post.kek.ip

hat@lanl.gov

walstrom@lanl.gov

twang@1anl.gov

twangler@1an1.gov

webber $@$,fnal.gov

weil@bnl.gov

\section{List of talks (http://www-bd.fnal.gov/icfa/snowmass/talks.html)}

\section{A. Overview:}

1. T. Wangler, High power proton linacs.

2. R. Macek, High intensity proton accumulators

3. F. Mills, High intensity proton synchrotrons

B. Machines - existing:

1. R. Shafer, LANSCE overview

2. D. Raparia, BNL $200 \mathrm{MeV}$ Linac

3. T. Roser, AGS and AGS Booster performance

4. R. Webber, Fermilab Booster performance and challenges

5. R. Cappi, High intensity issues in CERN PSB and PS

C. Machines - under construction:

1. J. Wei, Design and optimization of the SNS

2. S. Nath, SNS Linac

3. D. Raparia, SNS transfer lines

4. J. Galambos, SNS beam loss, activation and collimation

5. S. Machida, JHF project and lattice

D. Machines - proposed:

1. W. Chou, The Fermilab Proton Driver

2. T. Roser, $1 \mathrm{MW}$ AGS Proton Driver

3. H. Haseroth, CERN Proton Driver (SPL)

4. R. Cappi, CERN Proton Driver accumulator and compressor 
5. C. Prior, ESS and RAL Proton Driver

6. J-M. Lagniel, CONCERT project

7. A. Thiessen, The Advanced Hydrotest Facility (AHF) overview

8. P. Schwandt, LANL AHF lattice

9. T. Wangler, Proton linac for nuclear waste transmutation.

10. R. Johnson, Fermilab Linac Afterburner in the Booster tunnel

11. S. Machida, Progress on FFAG accelerators

12. S. Martin, The FFAG is a challenge

13. K. Takayama, Induction synchrotron

14. J-M: Lagniel, Challenges and R\&D for new facilities

15. S. Martin, Topics to study for making ESS less expensive

16. S. Martin, SC linac optimization

E. Accelerator physics and experiments:

1. C. Prior, Lattice, injection and space charge

2. S. Machidà, Space charge in rings

3. J. Holmes, Resonant beam response in the PSR accumulator ring

4. J. Holmes, Transverse impedance model for particle tracking calculations

5. I. Hofmann, Coulomb effects in high intensity drivers

6. I. Hofmann, Resonances in high intensity linacs (and rings)

7. R. Ryne, Simulation for high intensity linac and ring

8. H. Qin, Beam instabilities

9. F. Ruggiero, Collective and electron cloud effects at CERN SPS and LHC

10. M. Furman, Electron cloud and e-p instability

11. T-S. Wang, A chat about transverse e-p instability

12. Y. Shimosaki: Halo formation and equilibrium in high intensity hadron rings

13. P. Colestock, Beam halo formation in high-current proton linacs

14. N. Mokhov, Beam loss and shielding

15. S. Koscielniak, Beam loading and compensation

16. R. Baartman, End effects of beam transport elements

F. Accelerator systems:

1. K-N. Leung, High intensity negative ion sources

2. J. Griffin, RF system and inductive insert

3. F. Ostiguy, Proton driver magnets

4. P. Walstrom, Extraction kickers and modulators for the AHF

5. A. Drozhdin, Beam collimation in low and high-energy accelerators

6. R. Shafer, Diagnostics for high intensity hadron accelerators

7. R. Webber, Scope of proton driver beam diagnostics

G. M6 working group activity reports:

1. W. Chou: Report at the July 12 mid-term plenary session

2. W. Chou and J. Wei: Report at the July 20 final plenary session 